\title{
SUBSETS OF HARDY-CLASS ZERO IN THE BALL
}

\author{
PASCAL J. ThOMAS
}

\begin{abstract}
We consider the problem of whether a union of complex hyperplanes can be a subset of a zero variety for the Hardy classes of the ball. A sufficient condition is found, consisting in a strong geometric separatedness requirement, together with a quantitative requirement slightly stronger than the necessary condition for Nevanlinna class zero varieties.
\end{abstract}

\section{Introduction}

Context. This paper came out of conversations with Eric Amar on the subject of trying to find uniqueness sets for Hardy classes in the unit ball of $C^{n}$ made up of complex hyperplanes. A uniqueness set for $H^{p}\left(B^{n}\right), V \subset B^{n}$, is a set such that if $f \in H^{p}\left(B^{n}\right)$ and $f \equiv 0$ on $V$, then $f \equiv 0$. Mathematics being a devious game, what I actually found are large classes of examples of sets which are not uniqueness sets, that is to say that they are subsets of zero-sets, which are sets of the form $V^{\prime}=\{f=0\} \subset B^{n}$, with $f \in H^{p}\left(B^{n}\right)$ and $V^{\prime} \neq B^{n}$.

Notations. For $z, w, \in C^{n}$,

$$
\begin{gathered}
z \cdot \bar{w}=\sum_{i=1}^{n} z_{i} \bar{w}_{i} . \\
B^{n}=\left\{z \in C^{n}:|z|^{2}=z \cdot \bar{z}<1\right\} .
\end{gathered}
$$

For $a \in B^{n}, a \neq 0, a^{*}=\frac{a}{|a|}$.

We let $V=\bigcup_{j} V_{j}$ where the $V_{j}$ are hyperplanes defined by:

$$
V_{j}:=\left\{z \in B^{n}: z \cdot \bar{a}_{j}=\left|a_{j}\right|^{2}\right\} .
$$

The point $a_{j}$ is the point in $V_{j}$ closest to the origin. This notation excludes the exceptional case of a hyperplane through the origin.

Finally $H^{p}\left(B^{n}\right)$ is the space of functions $f$ holomorphic in $B^{n}$ such that $\|f\|_{p}^{p}:=\sup _{r<1} \int_{\partial B^{n}}|f(r \zeta)|^{p} d \sigma(\zeta)<\infty$, where $\sigma$ is the $2 n-1$-real-dimensional normalised Lebesgue measure on $\partial B^{n}$. 
Previous results. The only necessary and sufficient condition as far as zero-sets are concerned is due to Henkin and Skoda [6] and states that $V$ is a zero-set for a function in the Nevanlinna class (which contains all the Hardy classes) if and only if $V$ verifies the Blaschke condition, i.e. the integral of the distance to the boundary with respect to surface measure on $V$ is finite. Specialized to the case of a union of hyperplanes, this says

$$
\sum\left(1-\left|a_{k}\right|^{2}\right)^{n}<\infty
$$

Hakim and Sibony [2] proved that this is quantitatively sharp in the sense that given any decreasing function $\varphi(x)$ defined on $(0,1]$ and tending to infinity as $x$ tends to 0 , there exists a bounded holomorphic function $f$ such that on $V=\{f=0\}, \delta \varphi(\delta)$ has infinite integral, where $\delta$ stands for the distance to the boundary. That set $V$ is not a union of hyperplanes.

In the same direction, Berndtsson [1] found, given $\epsilon>0$, examples of zerosets for bounded functions made up of a union of hyperplanes verifying

$$
\sum\left(1-\left|a_{k}\right|^{2}\right)^{n-\epsilon}=\infty .
$$

The hyperplanes in Berndtsson's example verify in particular the following separatedness property: given a positive constant $\lambda>1$, if we call Tent around $a_{k}$ (and around the corresponding hyperplane) the set

$$
T_{\lambda}\left(a_{k}\right):=\left\{z \in \overline{B^{n}}:\left|1-z \cdot \bar{a}_{k}\right| \leq \lambda\left(1-\left|a_{k}\right|^{2}\right)\right\},
$$

then for $j \neq k, T_{\lambda}\left(a_{j}\right) \cap T_{\lambda}\left(a_{k}\right)=0$. Berndtsson's result can be obtained in the following way: for any $\epsilon>0$, set

$$
T_{\lambda}^{\epsilon}\left(a_{k}\right):=\left\{z \in \overline{B^{n}}:\left|I-z \cdot \bar{a}_{k}\right| \leq \lambda\left(1-\left|a_{k}\right|^{2}\right)^{1-\epsilon}\right\} .
$$

Then a union of hyperplanes verifying for $j \neq k, T_{\lambda}^{\epsilon}\left(a_{j}\right) \cap T_{\lambda}^{\epsilon}\left(a_{k}\right)=\emptyset$ is included in a zero set for a bounded function [7].

An obvious stufficient condition for $V$ to be a zero-set for a bounded function is

$$
\sum\left(1-\left|a_{k}\right|^{2}\right)<\infty,
$$

for one then can build a several dimensional analogue of a Blaschke product. Rudin, using a result of Shapiro and Shields [4, p. 135], [5], exhibited examples proving that no weaker quantitative condition can be sufficient: if the $a_{k}$ are all on the same real line and

$$
\sum\left(1-\left|a_{k}\right|^{2}\right)=\infty
$$

then $V$ is a uniqueness set for a class containing all the Hardy classes. Note that $H^{p}$-zero-sets are different for different values of $p$ in a very strong sense; see $[4, \mathrm{pp} .133-145]$ for this and many more details on the subject. 
The case where all the $a_{k}$ are on the same complex line corresponds to a family of parallel hyperplanes. To avoid this, we need to add some geometric separatedness condition to the quantitative condition to obtain a sufficient condition for a zero-set (or a subset of one). Varopotulos [8] gave a tuniform version of the Blaschke condition which is sufficient to get a zero-set for $H^{p}$ for some $p>0$. For unions of hyperplanes, the Uniform Blaschke condition translates into

(1) $\mu$ is a Carleson measure, where $\mu:=\sum_{j}\left(1-\left|a_{j}\right|^{2}\right)^{n} \delta_{a_{j}}$, and

(2) there are constants $C_{1}$ and $C_{2}$ such that

$$
\left\{j: V_{j} \nsubseteq T_{C_{2} h}(z)\right\} \leq C_{2} .
$$

(1) is equivalent to

$$
\sum_{j: a_{j} \in T_{\lambda}(a)}\left(1-\left|a_{j}\right|^{2}\right)^{n} \leq C \lambda^{n}\left(1-|a|^{2}\right)^{n}
$$

and (see [3] ) to

$$
\sup _{k} \sum_{j}\left(\frac{\left(1-\left|a_{k}\right|^{2}\right)\left(1-\left|a_{j}\right|^{2}\right)}{\left|I-a_{k} \cdot \overline{a_{j}}\right|^{2}}\right)^{n}<\infty
$$

In particular we see that the requirement that tents for different hyperplanes be disjoint is much stronger than the Uniform Blaschke condition, although both are quantitatively close to the Blaschke condition.

Varopoulos also proved in [8] that the Uniform Blaschke condition alone is not sufficient to get high values of $p$ : for any $p_{0}>0$, there exists a union $V$ of hyperplanes verifying the Uniform Blaschke condition which is a uniqueness set for $H^{p_{b}}$. In fact he points out that his theorem gives poor control on the value of $p$.

\section{Results and open questions}

We proceed to give sufficient conditions exploiting the disjointness of tents.

Theorem 1. If $V$ verifies that there exists a decreasing function $\varphi$ from $(0,1]$ to $R_{+}{ }^{*}$ and $A>0$ such that for $0<x<x_{0}, \varphi(x) \geq|\log x|^{A}$ and

$$
\sum_{k}\left(1-\left|a_{k}\right|^{2}\right)^{n} \varphi\left(1-\left|a_{k}\right|^{2}\right)<\infty
$$

and that there exists $\lambda_{0} \geq 9$ such that

$$
T_{\lambda_{\theta}\left(a_{j}\right) \cap T_{\lambda_{\theta}}\left(a_{k}\right)=\emptyset \text { for all } j \neq k}
$$


then there exists $F \in \bigcap_{p<p_{0}} H^{p}\left(B^{n}\right)$, with $p_{0}=C\left(\lambda_{0}\right) A$, such that $F \equiv 0$ on $V$ and $F$ is not identically zero.

Corollary 2. If assumption $\left(Q_{1}\right)$ is replaced by

$$
\sum_{k}\left(1-\left|a_{k}\right|^{2}\right)^{n-\epsilon}<\infty
$$

for some $\epsilon>0$, then $V$ is a subset of a zero set for $\bigcap_{p}>0 H^{p}\left(B^{n}\right)$.

Proof of Corollary 2: In the above, set $\varphi(x)=x^{-\epsilon}$. The constuction of $F$ (see below) does not depend on $p$, and $F \in \bigcap_{p>0} H^{p}\left(B^{n}\right)$.

Open Questions. One would like to obtain a bounded function instead of the intersection of the Hardy classes. Also it would be nice if $V$ could be shown to be exactly a zero set - the vanishing function $F$ being given by an infinite product, we can figure out $\{F=0\}$ explicitly. It is a union of hyperplanes too, much larger than $V$ even though it has roughly the same volume growth.

Proof of Theorem 1: We index $\left\{a_{k}\right\}$ so that $\left|a_{k}\right| \leq\left|a_{k+1}\right|$, and set

$$
F_{k}(z)=\left(\frac{1-\left|a_{k}\right|^{2}}{I-z \cdot \bar{a}_{k}}\right)^{N\left(\left|a_{k}\right|\right)}
$$

where $N(r)$ is a positive increasing function of $r$, with $N(0)>n . F_{k}$ is holomorphic on $B^{n}$ since $\operatorname{Re}\left(1-z \cdot \bar{a}_{k}\right)>0$ for $z \in B^{n}$.

$$
\sum\left|F_{k}(0)\right|=\sum_{k}\left(1-\left|a_{k}\right|^{2}\right)^{N\left(\left|\alpha_{k}\right|\right)}<\infty,
$$

therefore the following infinite product

$$
F(z):=\prod_{k=1}^{\infty}\left(1-F_{k}(z)\right)
$$

converges at least for $z=0$. In fact for $|z| \leq \gamma<1$, then

$$
\left|\frac{1-\left|a_{k}\right|^{2}}{1-z \cdot \vec{a}_{k}}\right|^{N\left(\left|a_{k}\right|\right)} \leq\left(\frac{1-\left|a_{k}\right|^{2}}{1-\gamma}\right)^{n},
$$

so we also have convergence on any compact subset of $B^{n}$, and $F \not \equiv 0$. Clearly if $z \in V$, then $F_{k}(z)=1$ for some $k$, thus $F(z)=0$.

Now we have to bound

$$
\int_{\partial B^{n}}|F(r \zeta)|^{p} d \sigma(\zeta)
$$

for $r<1$. Set $s=\sqrt{\lambda_{0}}-1 \geq 2$. For simplicity, we shall write $T\left(a_{j}\right)$ for $T_{\lambda_{0}}\left(a_{j}\right)$. 
Lemma 1. Under assumption ( $\left.S_{1}\right)$, if the function $N$ is chosen so that

$$
\alpha:=\sum_{m \geq 0} s^{-2 N\left(1-s^{-m-2}\right)}<\infty,
$$

then for any $z \in B^{n} \backslash \bigcup_{j \in A} T\left(a_{j}\right)$,

$$
\left|\prod_{j \in A}\left(1-F_{j}(z)\right)\right| \leq e^{C \alpha},
$$

where $A$ is an index set. (For example the complement of a singleton.)

The proofs of all lemmas are deferred until the end of the paper.

Using Lemma 1,

$$
\begin{aligned}
\int_{\partial B^{n}} \mid F(r \zeta)^{p} d \sigma(\zeta) & \\
& \leq \int_{r \zeta \notin \cup j, T\left(a_{j}\right)}|F(r \zeta)|^{p} d \sigma(\zeta)+\sum_{k} \int_{r \zeta \in T\left(a_{k}\right)}|F(r \zeta)|^{p} d \sigma(\zeta) \\
& \leq e^{C p \alpha} \sigma\left(\partial B^{n}\right)+e^{C p \alpha} \sum_{k} \int_{r \zeta \in T\left(a_{k}\right)}\left(1+\left|F_{k}(r \zeta)\right|\right)^{p} d \sigma(\zeta) \\
& \leq C_{p}\left(1+\sum_{k} \int_{r \zeta \in T\left(a_{k}\right)}\left|F_{k}(r \zeta)\right|^{p} d \sigma(\zeta)\right) \\
& \leq C_{p}\left(1+\sum_{k} \int_{\partial B^{n}}\left|F_{k}(r \zeta)\right|^{p} d \sigma(\zeta)\right)
\end{aligned}
$$

where $C_{p}$ is a constant depending on $n, \alpha$, and $p$, and we have used the fact that the tents are disjoint. We can bound each integral in the last sum by $\int_{\partial B^{n}}\left|F_{k}\right|^{p}$ since $F_{k}$ is holomorphic in a ball of radius $\left|a_{k}\right|^{-1}>1$. So we just have to estimate

$$
\begin{aligned}
\int_{\partial B^{n}} & \left(\frac{1-\left|a_{k}\right|^{2}}{1-\zeta \cdot \bar{a}_{k}}\right)^{p N\left(\left|a_{k}\right|\right)} d \sigma(\zeta) \\
& \leq 2^{p N\left(\left|a_{k}\right|\right)} \int_{\partial B^{n}}\left(\frac{1-\left|a_{k}\right|}{1-\zeta \cdot \bar{a}_{k}}\right)^{p N\left(\left|a_{k}\right|\right)} d \sigma(\zeta)
\end{aligned}
$$

and since the integrand is less than one and $N\left(\left|a_{k}\right|\right)>n$, this last integral is bounded by $C 2^{p N\left(\left|a_{k}\right|\right)}\left(1-\left|a_{k}\right|^{2}\right)^{n}$. The proof of the theorem will be concluded with: 
Lemma 2. For $p<p_{0}=2 A \log s / \log 2$, one can choose a function $N(r)$ as above so that

$$
\sum_{m \geq 0} s^{-2 N\left(1-s^{-m-2}\right)}<\infty
$$

and $2^{p N\left(\left|a_{k}\right|\right)} \leq \varphi\left(1-\left|a_{k}\right|^{2}\right)$ for $k$ large enough.

The method presented here does not allow us to weaken the separatedness condition to a Carleson-measure hypothesis, nor to render the value of $\lambda_{\mathfrak{a}}$ arbitrarily small. However we can prove:

Proposition 3. Theorem 1 still holds with $p_{0}$ replaced with $p_{0} / M$, ond therefore Corollary 2 holds unchanged, if we replace assumption $\left(S_{1}\right)$ with the weaker

$\left(S_{2}\right) \quad$ There is an integer $M$ such that for all $\zeta \in \partial B^{n}, \quad \sum_{j} \chi_{j}(\zeta) \leq M$ where $\chi_{j}:=\chi_{T\left(a_{j}\right)}$ is the indicator function of $T\left(a_{j}\right)$.

Proof: If $A$ is an index set, let $P_{A}=\bigcap_{j \in A} T\left(a_{j}\right) \cap \bigcap_{j \notin A} T\left(a_{j}\right)^{c}$. These sets are disjoint for different index sets. Under hypothesis $\left(S_{2}\right)$, we need only consider $P_{A}$ for $\# A \leq M$ (otherwise $P_{A}=$ ). Lemma 1 still holds under $\left(S_{2}\right)$, only a constant is changed in the estimation of $\# A_{m}^{l}$.

$$
\begin{aligned}
\int_{\partial B^{n}}|F(r \zeta)|^{p} d \sigma(\zeta) & =\sum_{A} \int_{r \zeta \in P_{A}}|F(r \zeta)|^{p} d \sigma(\zeta) \\
& \leq e^{C \alpha} \sum_{A} \int_{r \zeta \in P_{A}} \prod_{j \in A}\left|1-F_{j}(r \zeta)\right|^{p} d \sigma(\zeta)
\end{aligned}
$$

For $A=\emptyset$, we just integrate a constant over a subset of $\partial B^{n}$. For $A \neq \emptyset$, let $j_{A}=\max A$. For all $j$ and $z,\left|F_{j}(z)\right| \leq 2^{N\left(\left|a_{j}\right|\right)}$.

$$
\begin{aligned}
\prod_{j \in A}\left|1-F_{j}(r \zeta)\right| & \leq\left|1-F_{j_{A}}(r \zeta)\right| \prod_{j \in A \backslash\left(j_{A}\right\}}\left(1+2^{N\left(\left|a_{j}\right|\right)}\right) \\
& \leq 2^{M-1} 2^{(M-1) N\left(\left|a_{j_{A}}\right|\right)}\left|1-F_{j_{A}}(r \zeta)\right|
\end{aligned}
$$

Thus

$$
\begin{aligned}
& \sum_{A \neq \emptyset} \int_{r \zeta \in P_{A}} \prod_{j \in A}\left|1-F_{j}(r \zeta)\right|^{p} d \sigma(\zeta) \\
& \quad \leq C_{M, p} \sum_{A \neq \emptyset} 2^{p(M-1) N\left(\left|a_{j_{A}}\right|\right)} \int_{r \zeta \in P_{A}} \mid 1-F_{j_{A}}\left(\left.r \zeta\right|^{p} d \sigma(\zeta)\right. \\
& \quad \leq C_{M, p} \sum_{l \geq 1} 2^{p(M-1) N\left(\left|a_{i}\right|\right)} \int_{r \zeta \in T^{\prime}}\left|1-F_{l}(r \zeta)\right|^{p} d \sigma(\zeta)
\end{aligned}
$$


where $T_{l}^{\prime}:=\bigcup_{A: \max A=l} P_{A}=T\left(a_{i}\right) \backslash \bigcup_{k>l} T\left(a_{k}\right)$. Since $z \in T\left(a_{l}\right)$ if and only if $\left|F_{l}(z)\right| \geq \lambda_{0}^{-1}$, we have

$$
\begin{aligned}
\int_{r \zeta \in T^{\prime} l}\left|1-F_{l}(r \zeta)\right|^{p} d \sigma(\zeta) & \leq C_{p} \int_{r \zeta \in T^{\prime},}\left|F_{l}(r \zeta)\right|^{p} d \sigma(\zeta) \\
& \leq C_{p} \int_{\partial B^{n}}\left|F_{l}(\zeta)\right|^{p} d \sigma(\zeta) \\
& \leq C_{p} 2^{p\left(\left|a_{t}\right|\right)}\left(1-\left|a_{i}\right|^{2}\right)^{n}
\end{aligned}
$$

Finally we have to bound $\sum_{i} 2^{p M N\left(\left|a_{i}\right|\right)}\left(1-\left|a_{l}\right|^{2}\right)^{n}$, which is done by applying Lemma 2 with $p^{\prime}=M p$, whence the value $p_{0} / M$.

\section{Proof of the Lemmas}

\section{Proof of Lemma 1:}

We shall fix $z \in \overline{B^{n}}$ and estimate $F(z)$. Let $k \in A$, so that $z \notin T\left(a_{k}\right)$. By the triangle inequality for $|1-z \cdot \bar{w}|^{1 / 2}([4, \mathrm{p} .66])$ :

$$
\begin{aligned}
\left|1-z \cdot \bar{a}_{k}^{*}\right|^{1 / 2} & \geq \sqrt{\lambda_{0}}\left(1-\left|a_{k}\right|^{2}\right)^{1 / 2}-\left(1-\left|a_{k}\right|\right)^{1 / 2} \\
& =\left(1-\left|a_{k}\right|\right)^{1 / 2}\left(\sqrt{\lambda_{0}}\left(1+\left|a_{k}\right|\right)^{1 / 2}-1\right) \\
& >s\left(1-\left|a_{k}\right|\right)^{1 / 2}, \\
\text { i.e. } & \left|1-z \cdot \bar{a}_{k}^{*}\right|>s^{2}\left(1-\left|a_{k}\right|\right) .
\end{aligned}
$$

For $m \in Z_{+}$, let

$$
A_{m}=A_{m}(z)=\left\{a_{k}: s^{-m}<\left|1-z \cdot \bar{a}_{k}^{*}\right| \leq s^{-m+1}\right\} .
$$

Note that $\lambda_{0} \geq 9 \Rightarrow s \geq 2$ and $A_{m}$ would be empty for $m \leq-1$. For $a_{k} \in A_{m}$,

$$
1-\left|a_{k}\right|^{2} \leq s^{-2}\left|1-z \cdot \bar{a}_{k}^{*}\right| \leq s^{-m+1} .
$$

We must estimate

$$
\left|\prod_{k \notin A}\left(1-\left(\frac{1-\left|a_{k}\right|^{2}}{1-z \cdot \bar{a}_{k}}\right)^{N\left(\left|a_{k}\right|\right)}\right)\right| \leq \exp \sum_{m=0}^{\infty} \sum_{a_{k} \in A_{m}}\left(\frac{1-\left|a_{k}\right|^{2}}{\left|1-z \cdot \bar{a}_{k}\right|}\right)^{N\left(\left|a_{k}\right|\right)}
$$

Let us bound the inner sum. We always have

$$
\frac{1-\left|a_{k}\right|^{2}}{\left|1-z \cdot \bar{a}_{k}\right|} \leq \frac{1}{\lambda_{0}}=\frac{1}{(s+1)^{2}} \leq s^{-2} .
$$

Set

$$
A_{m}^{l}=A_{m} \cap\left\{a: 1-|a| \in\left(s^{-i-m-1}, s^{-i-m}\right]\right\} .
$$


Then $A_{m}=\bigcup_{l} A_{m}^{l}$, and if $a_{k} \in A_{m}^{l}$,

$$
\begin{aligned}
\frac{1-\left|a_{k}\right|^{2}}{\left|1-z \cdot \bar{a}_{k}\right|} & \leq \frac{1-\left|a_{k}\right|^{2}}{\left(\left|1-z \cdot \bar{a}_{k}^{*}\right|^{1 / 2}-\left(1-\left|a_{k}\right|^{2}\right)^{1 / 2}\right)^{2}} \\
& \leq \frac{2}{(1-1 / s)^{2}} \frac{1-\left|a_{k}\right|}{\left|1-z \cdot \bar{a}_{k}^{*}\right|} \leq s^{3-1},
\end{aligned}
$$

thus for all $a_{k} \in A_{m}^{l}, N\left(\left|a_{k}\right|\right) \leq N\left(1-s^{-i-m-1}\right)$ and

$$
\frac{1-\left|a_{k}\right|^{2}}{\left|1-z \cdot \bar{a}_{k}\right|} \leq \min \left(s^{-2}, s^{2-l}\right)
$$

Since the tents associated to different $a_{k}$ are disjoint,

$$
\left.\sum_{a_{k} \in A_{m}^{\dagger}} \sigma\left(T\left(a_{k}\right)\right) \leq \sigma\left(\zeta \in \partial B^{n}: \mid 1-\zeta \cdot \bar{a}_{k}^{*}\right\} \leq s^{-m+1}\right) \leq s^{(-m+1) n} .
$$

Since $\sigma\left(T\left(a_{k}\right)\right) \geq c_{n} \lambda_{0}{ }^{n} s^{-(m+l+1) n}$, we have $\# A_{m}^{l} \leq C_{n, \lambda_{0}} s^{l n}$. Finally

$$
\begin{aligned}
& \sum_{m=0}^{\infty} \sum_{a_{k} \in A_{m}}\left(\frac{1-\left|a_{k}\right|^{2}}{\left|1-z \cdot \bar{a}_{k}\right|}\right)^{N\left(\left|a_{k}\right|\right)} \\
& \quad \leq C \sum_{m=0}^{\infty} \sum_{l=1}^{\infty} s^{l n}\left[\min \left(s^{-2}, s^{3-l}\right)\right]^{N\left(1-s^{-l-m-1}\right)} \\
& \quad \leq C \sum_{m=0}^{\infty}\left[4 s^{-2 N\left(1-s^{-m-2}\right)}+s^{3} \sum_{l=3}^{\infty} s^{\left.l\left(n-N\left(1-s^{-i-m-2}\right)\right)\right]}\right.
\end{aligned}
$$

Since $N$ is increasing we can bound the inner series by a geometric one

$$
\leq C \sum_{m=0}^{\infty}\left(s^{-2 N\left(1-s^{-m-2}\right)}+\frac{s^{3\left(n-N\left(1-s^{-m-4}\right)\right)}}{1-s^{n-N\left(1-s^{-m-4}\right)}}\right) \text {. }
$$

Note that $s \geq 2$ and $N\left(1-s^{-m-4}\right) \geq N\left(1-s^{-4}\right)$; so, choosing $N$ so that $N\left(1-s^{-4}\right) \geq 3 n$, we finally majorize by

$$
C \sum_{m=0}^{\infty} s^{-2 N\left(1-s^{-m-2}\right)},
$$

a convergent sum by assumption.

Proof of Lemma 2:

For any given $k$, choose $m=m_{k}$ so that

$$
1-s^{-m-2} \leq\left|a_{k}\right|^{2}<1-s^{-m-3} .
$$


Set

$$
N\left(1-s^{-m-2}\right)=\frac{\log m+2 \log \log m}{2 \log s},
$$

and extend it as an increasing function on $[0,1)$. Then $s^{-2 N\left(1-s^{-m-2}\right)}=$ $1 / m(\log m)^{2}$, and the convergence of the first series is ensured.

On the other hand,

$$
\begin{gathered}
N\left(\left|a_{k}\right|\right)<N\left(1-s^{-m-3}\right)=\frac{1}{2 \log s}(\log (m+1)+2 \log \log (m+1)), \\
\quad \text { So } 2^{p N\left(\left|a_{k}\right|\right)} \leq(m+1)^{\frac{2 \log 2}{2 \log s}}(\log (m+1))^{\frac{p \log 2}{\log s}},
\end{gathered}
$$

and for $m$ large enough

$$
\varphi\left(1-\left|a_{k}\right|^{2}\right) \geq \varphi\left(s^{-m-2}\right) \geq(m+2)^{A}(\log s)^{A}
$$

For $p<p_{0}=2 A \log s / \log 2$, the second inequality in the Lemma 2 will be verified for $m=m_{k}$ large enough, thus for $k$ large enough.

\section{References}

1. Berndtsson, B., Interpolating sequences for $H^{\infty}$ in the ball, Proc. Kon. Ned. Akad. van Wetensch. A 88 (1985), 1-10.

2. HAKIM, M. AND Sibony, N., Ensemble des zéros d'une fonction holomorphe bornée dans la boule unité, Math. Ann. 260, 4 (1982), 469-474.

3. Mantero, A. M., Sur la condition de Carleson dans la boule unité de $C^{m}$, Boll. Un. Mat. Ital. A 6, 2 (1983), 163-169.

4. Rudin, W., "Function Theory in the Unit Ball of $C^{\text {n }}$, Springer Verlag, New York, 1980.

5. Shapiro, H. AND Shields, A. L., On the zeros of functions with finite Dirichlet integral, Math. Z. 80 (1962), 217-229.

6. SKODA, H., Valeurs au bord pour les solutions de l'opérateur d", et caractérisation des zéros des fonctions de la classe de Nevanlinna, Bull. Soc. Math. France 104 (1976), 225-299. 
7. Thомаs, P. I., Tents and Interpolating Sequences in the Unit Ball, in "Complex Analysis II, Proceedings, University of Maryland 1985-86," Lecture Notes 1276, 291-302, Springer-Verlag, Berlin Heidelberg, 1987.

8. VARopoulos, N. Th., Zeros of $H^{p}$ functions in several complex variables, Pacific J. Math. 88, 8 (1980), 189-246.

Keywords. Blaschke condition, Carleson measures, zero sets

\author{
Département de Mathématiques \\ Université Paul Sabatier \\ 31062 Toulouse \\ FRANCE
}

Rebut el 3 d'Abril de 1989 